\title{
Nonindependent continuous-time random walks
}

\author{
Miquel Montero* and Jaume Masoliver ${ }^{\dagger}$ \\ Departament de Física Fonamental, Universitat de Barcelona, Diagonal 647, E-08028 Barcelona, Spain
}

(Received 27 July 2007; revised manuscript received 21 September 2007; published 13 December 2007)

\begin{abstract}
The usual development of the continuous-time random walk (CTRW) assumes that jumps and time intervals are a two-dimensional set of independent and identically distributed random variables. In this paper, we address the theoretical setting of nonindependent CTRWs where consecutive jumps and/or time intervals are correlated. An exact solution to the problem is obtained for the special but relevant case in which the correlation solely depends on the signs of consecutive jumps. Even in this simple case, some interesting features arise, such as transitions from unimodal to bimodal distributions due to correlation. We also develop the necessary analytical techniques and approximations to handle more general situations that can appear in practice.
\end{abstract}

DOI: 10.1103/PhysRevE.76.061115

PACS number(s): 02.50.Ey, 02.50.Ga, 05.40.Fb, 02.30.Mv

\section{INTRODUCTION}

For more than four decades, since their introduction in 1965 by Montroll and Weiss [1], continuous-time random walks (CTRWs) have been applied to virtually any field in which one wishes to provide a dynamical description of the microstructure of a given random system. A huge number of examples and applications can be found in the literature, of which we cite only a handful: transport in disordered media [2,3], random networks [4], self-organized criticality [5], electron tunneling [6], earthquake modeling [7,8], hydrology $[9,10]$, time-series analysis $[11,12]$, and finance [13-21].

The CTRW generalizes the ordinary random walk since in the latter the steps of the random walker are made at equal intervals of time, while in the CTRW the interval between steps is a continuous random variable. In this sense, the CTRW is related to several other extensions of random walks in continuous time, like semi-Markov processes or Markov renewal processes [22], although the seeds of this idea can be traced back to the 1920s with the pure birth Poisson process $[23,24]$.

A great number of developments of the CTRW are based on the assumption that the magnitude of the steps (or jumps) and the time intervals between them (also called sojourns) are a two-dimensional set of independent and identically distributed random variables. While in many cases this is a convenient assumption which allows for simple developments, there are some other cases in which independent walks are clearly insufficient to explain some aspects of the physical reality, and correlations between consecutive step sizes and/or waiting times must be considered. We have met with such a case in our study of financial time series regarding their extreme time statistics [25], where jump magnitudes (transaction-to-transaction returns) show short-range memory, but it seems to be relevant also in earthquake modeling, where some evidence pointing to the presence of cross correlations between simultaneous and sequential earthquake magnitudes and recurrence times has been reported [26].

\footnotetext{
*miquel.montero@ub.edu

†jaume.masoliver@ub.edu
}

Successive recurrence times appear to be positively selfcorrelated as well but, oddly enough, consecutive magnitudes seem to be independent in this case.

Our goal in this paper is to address the theoretical setting of nonindependent CTRWs, a class of random walks that can account for many physical situations. In this kind of walk, jumps and sojourns are no longer independent random variables and their value at a given step may depend on previous steps. We develop the formalism for the Markovian case in which the magnitudes of a given jump and time interval depend only on the preceding step. We also obtain a complete solution to the problem when the correlation between consecutive steps solely depend on the sign of the previous jump (that is, whether the previous jumps is increasing or decreasing but not on its magnitude). Particular examples corresponding to this solvable case allow us to visualize and quantify some interesting consequences of the existence of correlations between steps as, for instance, the transitions from unimodal to bimodal distributions. Finally, we also develop the necessary perturbation techniques to deal with more general situations.

The paper is organized as follows. In Sec. II we outline the traditional CTRW based on the assumption of independence between events. In Sec. III we present the general setting for nonindependent CTRWs that are still amenable to analytical treatment. In Sec. IV we present an exact solution to the problem. Section V is devoted to considering weak dependent models for which we develop a complete perturbation technique. Conclusions are drawn in Sec. VI. Although this work is essentially technical, some even more technical aspects are in an Appendix.

\section{THE INDEPENDENT CTRW}

Suppose that a given random process $X(t)$ evolves following a CTRW. In this picture, any realization of $X(t)$ consists of a series of step functions, and $X(t)$ changes at random times $\ldots, t_{-2}, t_{-1}, t_{0}, t_{1}, t_{2}, \ldots$ while it remains fixed in place between successive steps. The interval between these successive steps is a random variable $\Delta t_{n}=t_{n}-t_{n-1}$, which we call the sojourn or waiting time. At the conclusion of the $n$th 
sojourn, $X(t)$ experiences a random change, or jump, given by

$$
\Delta X_{n}=\Delta X_{n}\left(\Delta t_{n}\right)=X\left(t_{n}\right)-X\left(t_{n-1}\right)=X_{n}-X_{n-1}
$$

In the usual model of the CTRW, waiting times $\Delta t_{n}$ and random jumps $\Delta X_{n}$ constitute a two-dimensional set of identically distributed random variables $\left(\Delta X_{n}, \Delta t_{n}\right)$, described by the corresponding joint probability density function (PDF) $\rho(\xi, \tau)$,

$$
\rho(\xi, \tau) d \xi d \tau=\operatorname{Prob}\left\{\xi<\Delta X_{n} \leqslant \xi+d \xi ; \tau<\Delta t_{n} \leqslant \tau+d \tau\right\} .
$$

As usual, two marginal PDFs can be derived from $\rho(\xi, \tau)$

$$
h(\xi) d \xi=\operatorname{Prob}\left\{\xi<\Delta X_{n} \leqslant \xi+d \xi\right\}
$$

and

$$
\psi(\tau) d \tau=\operatorname{Prob}\left\{\tau<\Delta t_{n} \leqslant \tau+d \tau\right\}
$$

just by integrating over the opposite variable:

$$
h(\xi)=\int_{0}^{\infty} \rho(\xi, \tau) d \tau, \quad \psi(\tau)=\int_{-\infty}^{\infty} \rho(\xi, \tau) d \xi
$$

In this setup, each pair of random variables $\left(\Delta X_{n}, \Delta t_{n}\right)$ is independent of any other pair $\left(\Delta X_{m}, \Delta t_{m}\right), m \neq n$, but any degree of correlation is still allowed between $\Delta X_{n}$ and $\Delta t_{n}$ themselves. 'Some CTRW processes belonging to this category can be found in [17-21], for instance, and sometimes are also named non independent models [19], since the label independent is then reserved for the particular case in which $\Delta X_{n}$ and $\Delta t_{n}$ are also mutually independent random variables, i.e., when

$$
\rho(\xi, \tau)=h(\xi) \psi(\tau)
$$

This divergence in the existing notation may therefore lead to misinterpretation. We prefer to call Eq. (2) the fully independent CTRW, and keep the term independent CTRW for any memoryless process with arbitrary joint PDF.

The chief objective of the CTRW formalism is to obtain the probability density function of $X(t)$. This PDF, called the propagator, is defined by

$$
p(x, t) d x=\operatorname{Prob}\left\{x<X(t) \leqslant x+d x \mid X\left(t_{0}\right)=0\right\},
$$

where in what follows we shall assume that the initial jump occurred at $t_{0}=0$. As is well known, the propagator obeys the following renewal equation [18]:

\footnotetext{
${ }^{1}$ From a mathematical point of view, this implies that $X(t)=X_{N(t)}$, $N(t)=\sup \left\{n \mid t_{n} \leqslant t\right\}$, can be thought of as a generalization of a semiMarkov process, since $X_{n}$ is, in general, not a Markov chain but a Markov discrete process with continuous states, and $\Delta t_{n}$ is a random variable depending on both $X_{n}$ and $X_{n-1}$ through $\Delta X_{n}$.
}

$$
p(x, t)=p_{0}(x, t)+\int_{0}^{t} d t^{\prime} \int_{-\infty}^{\infty} \rho\left(x-x^{\prime}, t-t^{\prime}\right) p\left(x^{\prime}, t^{\prime}\right) d x^{\prime} .
$$

The function $p_{0}(x, t)$ is the propagator prior to the first jump and, since the trajectories of $X(t)$ consist of a series of step functions, we write

$$
p_{0}(x, t)=\Psi(t) \delta(x),
$$

where $\Psi(t)$ is the probability that no transaction has occurred before time $t$,

$$
\Psi(t)=\int_{t}^{\infty} \psi\left(t^{\prime}\right) d t^{\prime}
$$

We can solve Eq. (3) in terms of the joint Fourier-Laplace transform

$$
\tilde{p}(\omega, s)=\int_{0}^{\infty} d t e^{-s t} \int_{-\infty}^{\infty} e^{i \omega t} p(x, t) d x .
$$

The solution is

$$
\tilde{p}(\omega, s)=\frac{\widetilde{p}_{0}(\omega, s)}{1-\tilde{\rho}(\omega, s)},
$$

where $\tilde{p}_{0}(\omega, s)$ and $\tilde{\rho}(\omega, s)$ are the joint Fourier-Laplace transforms of the functions $p_{0}(x, t)$ and $\rho(x, t)$. We easily see from Eqs. (4) and (5) that the explicit form of $\widetilde{p}_{0}(\omega, s)$ is

$$
\tilde{p}_{0}(\omega, s)=\frac{1-\hat{\psi}(s)}{s},
$$

where $\hat{\psi}(s)$ is the Laplace transform of the pausing-time density $\psi(\tau)$.

\section{A GENERAL NONINDEPENDENT WALK}

As we have explained, the CTRW outlined in the preceding section relies on the assumption that the pairs $\left(\Delta X_{n}, \Delta t_{n}\right)$ are independent two-dimensional random variables. However, as we mentioned in Sec. I, there are many situations in which the assumption of independence may be doubtful $[25,26]$. We shall thus generalize the CTRW formalism in order to account for memory effects due to correlations between different sojourns and/or jump increments.

Among the many ways of doing this extension, we choose a simple, but yet general, method which consists in assuming that the joint density of $k$ consecutive changes, $\rho\left(\xi_{n}, \tau_{n} ; \xi_{n-1}, \tau_{n-1} ; \ldots ; \xi_{n-k}, \tau_{n-k}\right)$, satisfies the Markov property

$$
\begin{aligned}
& \rho\left(\xi_{n}, \tau_{n} ; \xi_{n-1}, \tau_{n-1} ; \ldots ; \xi_{n-k}, \tau_{n-k}\right) \\
& \quad=\prod_{m=0}^{k-1} \rho\left(\xi_{n-m}, \tau_{n-m} \mid \xi_{n-m-1}, \tau_{n-m-1}\right) \rho\left(\xi_{n-k} \tau_{n-k}\right),
\end{aligned}
$$

where 


$$
\begin{aligned}
\rho\left(\xi^{\prime}, \tau^{\prime} \mid \xi, \tau\right) d \xi^{\prime} d \tau^{\prime}= & \operatorname{Prob}\left\{\xi^{\prime}<\Delta X_{n} \leqslant \xi^{\prime}+d \xi^{\prime} ;\right. \\
& \times \tau^{\prime}<\Delta t_{n} \leqslant \tau^{\prime}+d \tau^{\prime} \mid \Delta X_{n-1}=\xi ; \\
& \left.\times \Delta t_{n-1}=\tau\right\} .
\end{aligned}
$$

This means that the pair $\left(\Delta X_{n}, \Delta t_{n}\right)$ depends on all the previous transitions only through the immediately preceding one $\left(\Delta X_{n-1}, \Delta t_{n-1}\right)$. In an analogous way to the independent walk described by Eq. (3), the integral equation governing the evolution of the return PDF is given in this case by the renewal equation

$$
\begin{aligned}
p(x, t \mid \xi, \tau)= & p_{0}(x, t \mid \xi, \tau)+\int_{-\infty}^{\infty} d x^{\prime} \int_{0}^{t} \rho\left(x^{\prime}, t^{\prime} \mid \xi, \tau\right) \\
& \times p\left(x-x^{\prime}, t-t^{\prime} \mid x^{\prime}, t^{\prime}\right) d t^{\prime}
\end{aligned}
$$

where $p_{0}(x, t \mid \xi, \tau)$ is the propagator prior to the first jump, and similarly to Eq. (4) we write

$$
p_{0}(x, t \mid \xi, \tau)=\delta(x) \Psi(t \mid \xi, \tau)
$$

where $\Psi(t \mid \xi, \tau)$ is the cumulative distribution of the waiting time and is related to the transition density $\rho\left(x^{\prime}, t^{\prime} \mid \xi, \tau\right)$ by

$$
\Psi(t \mid \xi, \tau)=\int_{t}^{\infty} d t^{\prime} \int_{-\infty}^{\infty} \rho\left(x^{\prime}, t^{\prime} \mid \xi, \tau\right) d x^{\prime}
$$

Observe that in this case the process $X(t)$ is not Markovian because its PDF $p(x, t \mid \xi, \tau)$ depends on both the magnitude of the previous jump $\xi=X_{0}-X_{-1}$ and its sojourn time $\tau=t_{0}-t_{-1}$; in other words, the probability distribution of the process at a given time depends on two previous times $t_{0}$ and $t_{-1}$. We should note at this point that even the independent CTRW is, in general, a non-Markovian process. The only case in which the independent CTRW is Markovian is when it is a fully independent CTRW, and the set of random times $\ldots, t_{0}, t_{1}, t_{2}, \ldots$ is Poissonian, that is, when the pausing-time density $\psi(\tau)$ obeys the exponential law [3]

$$
\psi(\tau)=\lambda e^{-\lambda \tau} \quad(\lambda>0) .
$$

Let us remark that the dependent CTRW outlined above is always non-Markovian even for this Poissonian density.

We also note that in the case of independent increments discussed in Sec. II we have

$$
\rho\left(x^{\prime}, t^{\prime} \mid \xi, \tau\right)=\rho\left(x^{\prime}, t^{\prime}\right)
$$

and Eq. (8) reduces to Eq. (3).

The integral equation given in Eq. (8) is the general equation that governs the evolution of the random process $X(t)$ and it must be solved if we want to obtain the propagator for this nonindependent case. Contrary to the independent case, Eq. (8) cannot be solved for any form of the joint density $\rho$ by means of transform methods. Indeed, the Laplace transform with respect to $t$ of Eq. (8) is

$$
\begin{aligned}
\hat{p}(x, s \mid \xi, \tau)= & \hat{p}_{0}(x, s \mid \xi, \tau)+\int_{0}^{\infty} d t e^{-s t} \int_{0}^{t} d t^{\prime} \int_{-\infty}^{\infty} \rho\left(x^{\prime}, t^{\prime} \mid \xi, \tau\right) \\
& \times p\left(x-x^{\prime}, t-t^{\prime} \mid x^{\prime}, t^{\prime}\right) d x^{\prime}
\end{aligned}
$$

where the caret over $p$ and $p_{0}$ denotes the time Laplace transform. Note that

$$
\int_{0}^{\infty} d t e^{-s t} \int_{0}^{t} d t^{\prime} \cdots=\int_{0}^{\infty} d t^{\prime} e^{-s t^{\prime}} \int_{0}^{\infty} d t^{\prime \prime} e^{-s t^{\prime \prime}} \cdots
$$

where we have exchanged the order of integration and performed the change of variables $t^{\prime \prime}=t-t^{\prime}$. Then

$$
\begin{aligned}
\hat{p}(x, s \mid \xi, \tau)= & \hat{p}_{0}(x, s \mid \xi, \tau)+\int_{0}^{\infty} d t^{\prime} e^{-s t^{\prime}} \int_{-\infty}^{\infty} \rho\left(x^{\prime}, t^{\prime} \mid \xi, \tau\right) \\
& \times \hat{p}\left(x-x^{\prime}, s \mid x^{\prime}, t^{\prime}\right) d x^{\prime} .
\end{aligned}
$$

Finally, the Fourier transform with respect to $x$ of this equation yields

$$
\begin{aligned}
\tilde{p}(\omega, s \mid \xi, \tau)= & \tilde{p}_{0}(\omega, s \mid \xi, \tau)+\int_{0}^{\infty} d t^{\prime} e^{-s t^{\prime}} \int_{-\infty}^{\infty} e^{i \omega x^{\prime}} \rho\left(x^{\prime}, t^{\prime} \mid \xi, \tau\right) \\
& \times \widetilde{p}\left(\omega, s \mid x^{\prime}, t^{\prime}\right) d x^{\prime}
\end{aligned}
$$

which is the farthest we can go without specifying $\rho\left(x^{\prime}, t^{\prime} \mid \xi, \tau\right)$.

\section{A SOLVABLE CASE}

The integral equation (11) cannot be solved for any arbitrary form of $\rho$. However, for the independent case in which $\rho\left(x^{\prime}, t^{\prime} \mid \xi, \tau\right)=\rho\left(x^{\prime}, t^{\prime}\right)$ and $\tilde{p}\left(\omega, s \mid x^{\prime}, t^{\prime}\right)=\tilde{p}(\omega, s)$, we recover from Eq. (11) the solution given by Eq. (6). Another case in which the level of difficulty is somewhat reduced is when waiting times and jumps are not related to each other. In such a case, the joint density factorizes as

$$
\rho\left(x^{\prime}, t^{\prime} \mid \xi, \tau\right)=\psi\left(t^{\prime} \mid \xi, \tau\right) h\left(x^{\prime} \mid \xi, \tau\right) .
$$

We will also assume the further simplification

$$
\rho\left(x^{\prime}, t^{\prime} \mid \xi, \tau\right)=\psi\left(t^{\prime}\right) h\left(x^{\prime} \mid \xi\right)
$$

in which correlations between consecutive waiting times have been neglected, and we have also assumed that the waiting-time $\operatorname{PDF} \psi\left(t^{\prime}\right)$ does not depend on the magnitude of the jumps - a situation that, as mentioned above, has been detected in some financial time series [25].

The renewal equation for the propagator of $X(t)$ now reduces to [cf. Eqs. (8) and (9)]

$$
\begin{aligned}
p(x, t \mid \xi)= & \delta(x) \Psi(t)+\int_{0}^{t} d t^{\prime} \psi\left(t^{\prime}\right) \\
& \times \int_{-\infty}^{\infty} h\left(x^{\prime} \mid \xi\right) p\left(x-x^{\prime}, t-t^{\prime} \mid x^{\prime}, t^{\prime}\right) d x^{\prime} .
\end{aligned}
$$

The (time) Laplace transform of this equation yields 


$$
\hat{p}(x, s \mid \xi)=\delta(x) \hat{\Psi}(s)+\hat{\psi}(s) \int_{-\infty}^{\infty} h\left(x^{\prime} \mid \xi\right) \hat{p}\left(x-x^{\prime}, s \mid x^{\prime}\right) d x^{\prime},
$$

where, in terms of the density $\hat{\psi}(s)$, the waiting-time distribution function $\hat{\Psi}(s)$ can be written as

$$
\hat{\Psi}(s)=\frac{1-\hat{\psi}(s)}{s} .
$$

Let us now suppose that the conditional jump density $h\left(x^{\prime} \mid \xi\right)$ has the form

$$
h\left(x^{\prime} \mid \xi\right)=h\left(x^{\prime}\right)\left[1+\epsilon g\left(x^{\prime} \mid \xi\right)\right],
$$

where $\epsilon$ is an arbitrary parameter and $h\left(x^{\prime}\right)$ is the unconditional jump density—cf. Eq. (1)—which is related to $h\left(x^{\prime} \mid \xi\right)$ by the constraint

$$
h\left(x^{\prime}\right)=\int_{-\infty}^{\infty} h\left(x^{\prime} \mid \xi\right) h(\xi) d \xi .
$$

Note that this constraint prevents us from considering arbitrary functional forms for $h\left(x^{\prime} \mid \xi\right)$ and $h\left(x^{\prime}\right)$. For instance, if we set $h\left(x^{\prime} \mid \xi\right)=\left[\delta\left(x^{\prime}-\lambda \xi\right)+\delta\left(x^{\prime}+\lambda \xi\right)\right] / 2, \lambda \neq 1$, as in the case of the random walker with shrinking step sizes [27], Eq. (15) implies $h\left(x^{\prime}\right)=\delta\left(x^{\prime}\right)$. The distinctive point here is that, unlike geometric random walks, our (unconditional) PDF of $\Delta X_{n}$ does not depend on $n$.

The function $g\left(x^{\prime} \mid \xi\right)$ represents the correlation between previous and current jumps and $\epsilon$ governs its strength. Note that in order to meet Eq. (15) together with the normalization

$$
\int_{-\infty}^{\infty} h\left(x^{\prime} \mid \xi\right) d x^{\prime}=1 \text { for all } \xi,
$$

the correlation $g\left(x^{\prime} \mid \xi\right)$ must satisfy (see next section for a general discussion on this issue)

$$
\int_{-\infty}^{\infty} h\left(x^{\prime}\right) g\left(x^{\prime} \mid \xi\right) d x^{\prime}=\int_{-\infty}^{\infty} g\left(x^{\prime} \mid \xi\right) h(\xi) d \xi=0 .
$$

With the form of $h\left(x^{\prime} \mid \xi\right)$ given in Eq. (14) the integral equation for the propagator, Eq. (13), reads

$$
\begin{aligned}
\hat{p}(x, s \mid \xi)= & \delta(x) \hat{\Psi}(s)+\hat{\psi}(s) \int_{-\infty}^{\infty} h\left(x^{\prime}\right)\left[1+\epsilon g\left(x^{\prime} \mid \xi\right)\right] \\
& \times \hat{p}\left(x-x^{\prime}, s \mid x^{\prime}\right) d x^{\prime} .
\end{aligned}
$$

We will solve this equation for any even jump density,

$$
h\left(x^{\prime}\right)=h\left(-x^{\prime}\right),
$$

and when the correlation function has the following form:

$$
g\left(x^{\prime} \mid \xi\right)=\frac{x^{\prime} \xi}{\left|x^{\prime}\right||\xi|}=\operatorname{sgn}\left(x^{\prime}\right) \operatorname{sgn}(\xi),
$$

meaning that the dependence between current and previous jumps is only through their signs. In other words, the correlation depends on whether consecutive jumps are increasing or decreasing but not on their magnitude. From Eq. (14) we see that, in this case, since $h\left(x^{\prime} \mid \xi\right)$ must be positive definite, $-1 \leqslant \epsilon \leqslant 1$. In fact, this model might be interpreted as the simplest persistent CTRW [3], in which the probability that the process does not change its direction of movement is equal to $(1+\epsilon) / 2$, but where neither jumps nor sojourns are affected by this persistence. This is the kind of memory we adopted in [25] in order to model the observed anticorrelation, with origin in the bid-ask bounce effect: tick-by-tick price changes tend to oscillate back and forth between two values due to the bid-ask spread.

Let us note that the functional form of $g\left(x^{\prime} \mid \xi\right)$ given in Eq. (18) implies that any dependence on $\xi$ is only through $\operatorname{sgn}(\xi)=\xi /|\xi|$. Thus $p(x, t \mid \xi)=p(x, t \mid \operatorname{sgn}(\xi))$, which allows us to write

$$
p(x, t \mid \xi)=p^{(+)}(x, t) \Theta(\xi)+p^{(-)}(x, t) \Theta(-\xi),
$$

where $\Theta(\xi)$ is the Heaviside step function. The substitution of Eq. (19) into Eq. (16) yields for $\hat{p}^{( \pm)}(x, s)$ the following set of coupled integral equations:

$$
\begin{aligned}
\hat{p}^{(+)}(x, s)= & \delta(x) \hat{\Psi}(s)+(1+\epsilon) \hat{\psi}(s) \int_{0}^{\infty} h\left(x^{\prime}\right) \hat{p}^{(+)}\left(x-x^{\prime}, s\right) d x^{\prime} \\
& +(1-\epsilon) \hat{\psi}(s) \int_{-\infty}^{0} h\left(x^{\prime}\right) \hat{p}^{(-)}\left(x-x^{\prime}, s\right) d x^{\prime}, \\
\hat{p}^{(-)}(x, s)= & \delta(x) \hat{\Psi}(s)+(1-\epsilon) \hat{\psi}(s) \int_{0}^{\infty} h\left(x^{\prime}\right) \hat{p}^{(+)}\left(x-x^{\prime}, s\right) d x^{\prime} \\
& +(1+\epsilon) \hat{\psi}(s) \int_{-\infty}^{0} h\left(x^{\prime}\right) \hat{p}^{(-)}\left(x-x^{\prime}, s\right) d x^{\prime} .
\end{aligned}
$$

Now the Fourier transform with respect to $x$ turns this set into a system of algebraic equations:

$$
\begin{aligned}
\widetilde{p}^{(+)}(\omega, s)= & \hat{\Psi}(s)+\hat{\psi}(s)\left[(1+\epsilon) \tilde{H}(\omega) \widetilde{p}^{(+)}(\omega, s)\right. \\
& \left.+(1-\epsilon) \widetilde{H}(-\omega) \widetilde{p}^{(-)}(\omega, s)\right], \\
\widetilde{p}^{(-)}(\omega, s)= & \hat{\Psi}(s)+\hat{\psi}(s)\left[(1-\epsilon) \widetilde{H}(\omega) \widetilde{p}^{(+)}(\omega, s)\right. \\
& \left.+(1+\epsilon) \widetilde{H}(-\omega) \widetilde{p}^{(-)}(\omega, s)\right],
\end{aligned}
$$

where

$$
\widetilde{H}(\omega) \equiv \int_{0}^{\infty} e^{i \omega x^{\prime}} h\left(x^{\prime}\right) d x^{\prime}
$$

is the "half" Fourier transform of $h(x)$. Obviously,

$$
\tilde{h}(\omega)=\tilde{H}(\omega)+\widetilde{H}(-\omega),
$$

where $\widetilde{h}(\omega)$ is the "complete" Fourier transform of $h(x)$. We note that in writing Eqs. (20) and (21) and Eq. (22) we have imposed the symmetry of $h(x)$ assumed in Eq. (17).

Solving for Eqs. (20) and (21), we have 


$$
\widetilde{p}^{( \pm)}(\omega, s)=\frac{1-2 \epsilon \hat{\psi}(s) \tilde{H}(\mp \omega)}{1-(1+\epsilon) \hat{\psi}(s) \widetilde{h}(\omega)+4 \epsilon \hat{\psi}^{2}(s)|\widetilde{H}(\omega)|^{2}} \hat{\Psi}(s),
$$

where we have used the fact that for real jump densities $h(x)$ the identity $\widetilde{H}(\omega) \tilde{H}(-\omega)=|\widetilde{H}(\omega)|^{2}$ holds. The final solution to the problem is thus given by the combination of Eqs. (19) and (23). Therefore, under the assumptions given in Eqs. (12) and (14) and the special form of the correlation given in Eq. (18), we have been able to obtain an exact expression for the Fourier-Laplace transform of the propagator valid for any forms of the waiting-time density $\psi(\tau)$ and jump density $h(\xi)$, provided that the latter is an even function of $\xi$ with no bias.

Another interesting quantity is the unconditional propagator $p(x, t)$ defined by

$$
p(x, t)=\int_{-\infty}^{\infty} p(x, t \mid \xi) h(\xi) d \xi .
$$

In the analyzed case in which $p(x, t \mid \xi)$ can be decomposed as in Eq. (19) we have

$$
p(x, t)=\frac{1}{2}\left[p^{(+)}(x, t)+p^{(-)}(x, t)\right] .
$$

From Eqs. (23) and (24) we get

$$
\tilde{p}(\omega, s)=\frac{1-\epsilon \hat{\psi}(s) \tilde{h}(\omega)}{1-(1+\epsilon) \hat{\psi}(s) \tilde{h}(\omega)+4 \epsilon \hat{\psi}^{2}(s)|\widetilde{H}(\omega)|^{2}} \hat{\Psi}(s) .
$$

Note, incidentally, that when $\epsilon=0$ this expression reduces to

$$
\tilde{p}(\omega, s)=\frac{\hat{\Psi}(s)}{1-\hat{\psi}(s) \tilde{h}(\omega)},
$$

which agrees with the solution of the independent case discussed in Sec. II [cf. Eq. (6)].

Aside from the unconditional PDF $p(x, t)$, which provides maximal information about the evolution of $X(t)$, there is another quantity of considerable practical interest: the (unconditional) variance of $X(t)$. This quantity has the advantage that it does not require the knowledge of the entire jump distribution $h(\xi)$. It suffices to know the PDF $\psi(\tau)$ and the following two moments of $h(\xi)$ :

$$
\mu_{1} \equiv \int_{-\infty}^{\infty}|\xi| h(\xi) d \xi \quad \text { and } \quad \mu_{2} \equiv \int_{-\infty}^{\infty} \xi^{2} h(\xi) d \xi .
$$

Let $\left\langle X^{2}(t)\right\rangle$ be the unconditional second moment of the process:

$$
\left\langle X^{2}(t)\right\rangle=\int_{-\infty}^{\infty} x^{2} p(x, t) d x,
$$

and let us denote by $\hat{m}_{2}(s)$ its Laplace transform

$$
\hat{m}_{2}(s) \equiv \int_{0}^{\infty} e^{-s t}\left\langle X^{2}(t)\right\rangle d t .
$$

This can be written in terms of the joint Fourier-Laplace transform of $p(x, t)$ by

$$
\hat{m}_{2}(s)=-\left.\frac{\partial^{2} \widetilde{p}(\omega, s)}{\partial \omega^{2}}\right|_{\omega=0} .
$$

Recall that a direct consequence of the unbiased assumption expressed in Eq. (17) is that the odd moments of $h(\xi)$ are equal to zero. This implies that all odd moments of process $X(t)$ vanish as well; in particular, this means that the variance of $X(t)$ coincides with its second moment. The combination of Eqs. (25) and (26) leads, after some manipulations, to the relation

$$
\hat{m}_{2}(s)=\mu_{2} \frac{\hat{\psi}(s)}{s[1-\hat{\psi}(s)]}+2 \epsilon \mu_{1}^{2} \frac{\hat{\psi}^{2}(s)}{s[1-\hat{\psi}(s)][1-\epsilon \hat{\psi}(s)]} .
$$

Let us return to the propagator and particularize to the case of Poissonian waiting times for which $\psi(\tau)=\lambda e^{-\lambda \tau}$ and

$$
\hat{\psi}(s)=\frac{\lambda}{\lambda+s}, \quad \hat{\Psi}(s)=\frac{1}{\lambda+s} .
$$

Now Eq. (25) reads

$$
\tilde{p}(\omega, s)=\frac{\lambda+s-\lambda \epsilon \tilde{h}(\omega)}{(\lambda+s)^{2}-\lambda(1+\epsilon)(\lambda+s) \tilde{h}(\omega)+4 \lambda^{2} \epsilon|\tilde{H}(\omega)|^{2}},
$$

whose inverse Laplace transform yields the unconditional characteristic function [28]

$$
\begin{aligned}
\tilde{p}(\omega, t)= & e^{-\lambda t[1-(1+\epsilon) \tilde{h}(\omega) / 2]}(\cosh [\lambda t \tilde{k}(\omega) / 2] \\
& \left.+(1-\epsilon) \frac{\tilde{h}(\omega)}{\widetilde{k}(\omega)} \sinh [\lambda t \tilde{k}(\omega) / 2]\right),
\end{aligned}
$$

where

$$
\widetilde{k}(\omega) \equiv \sqrt{(1+\epsilon)^{2} \widetilde{h}^{2}(\omega)-16 \epsilon|\widetilde{H}(\omega)|^{2}} .
$$

As far as the second moment is concerned, Eq. (27) can be inverted at once with the result

$$
\left\langle X^{2}(t)\right\rangle=\mu_{2} \lambda t+\frac{2 \mu_{1}^{2} \epsilon}{(1-\epsilon)^{2}}\left[\lambda(1-\epsilon) t+e^{-\lambda(1-\epsilon) t}-1\right] .
$$

Observe that in the independent case $(\epsilon=0)$ the variance shows an ordinary diffusion behavior, while correlations introduce a richer dynamics.

In order to invert Eq. (30) and thus obtain an expression for the propagator $p(x, t)$, we must choose a functional form for the jump density $h(\xi)$. We will select the two-sided exponential density 


$$
h(\xi)=(\gamma / 2) e^{-\gamma|\xi|}
$$

for two main reasons. On the one hand it can be of interest in finance, the field that motivated this work in the first instance. Even though it is well established that PDFs of financial returns show a power-law decay [29], there is an increasing amount of evidence pointing to the fact that small and moderate returns are better described through a Laplace law-see [30] and references therein. On the other hand, one of the main motivations of this section is the introduction and subsequent analysis of a case for which we can obtain closed expressions. It is clear from Eq. (30) that when the characteristic function of $h(\xi)$ is intricate this goal will be well out of reach. In our case $\tilde{h}(\omega)$ is the inverse of a polynomial:

$$
\tilde{h}(\omega)=\frac{\gamma^{2}}{\gamma^{2}+\omega^{2}}, \quad \tilde{H}(\omega)=\frac{\gamma / 2}{\gamma-i \omega} .
$$

We incidentally note that now the variance of the process is given by Eq. (32) where $\mu_{1}=1 / \gamma$ and $\mu_{2}=2 / \gamma^{2}$. When 0 $<\epsilon \leqslant 1$ one can show that the Fourier inversion of Eq. (30) reads $^{2}$

$$
\begin{aligned}
p(x, t)= & e^{-\lambda t} \delta(x)+\frac{\gamma e^{-\lambda t}}{2 \sqrt{\epsilon}} \int_{\lambda \gamma|x| \sqrt{\epsilon}}^{\infty}\left(\frac{t}{u}\right)^{1 / 2} e^{-(1+\epsilon) u / 2 \lambda \epsilon}\left[I_{1}(2 \sqrt{u t})\right. \\
& \left.-\lambda \epsilon\left(\frac{t}{u}\right)^{1 / 2} I_{2}(2 \sqrt{u t})\right] I_{0}\left(\frac{(1-\epsilon)}{2 \lambda \epsilon} \sqrt{u^{2}-\epsilon \lambda^{2} \gamma^{2}|x|^{2}}\right) d u
\end{aligned}
$$

$(0<\epsilon \leqslant 1)$, where $I_{n}(z)$ are modified Bessel functions. Although the case $\epsilon=1$ is contained in Eq. (34) it can be written more explicitly as

$$
p(x, t)=e^{-\lambda t} \delta(x)+\frac{\gamma}{2} \sqrt{\frac{\lambda t}{\gamma|x|}} I_{1}(2 \sqrt{\gamma|x| \lambda t}) e^{-\lambda t-\gamma|x|} .
$$

The recovery of the independent case $\epsilon=0$ from Eq. (34) is a delicate issue that deserves special treatment. In this case, one has

$$
\begin{aligned}
p(x, t)= & e^{-\lambda t} \delta(x)+\gamma\left(\frac{\lambda t}{\pi}\right)^{1 / 2} \int_{0}^{\infty} \frac{I_{1}(u)}{u} \\
& \times \exp \left(-\frac{u^{2}}{4 \lambda t}-\frac{\gamma^{2}|x|^{2} \lambda t}{u^{2}}-\lambda t\right) d u
\end{aligned}
$$

$(\epsilon=0)$.

We can now graphically explore some of the most relevant properties of the unconditional propagator in the analyzed example. However, as we will show, our example will share those traits with any process that presents Poissonian waiting times, i.e., for which Eq. (32) stands. In Fig. 1 we plot the regular part of the probability density function (in $\gamma$ units) for different values of $\epsilon$. In particular, we present the

\footnotetext{
${ }^{2}$ In order to get Eqs. (34)-(36), as well as all the numerical results presented below, it is somewhat simpler to start from Eq. (29) and use Eq. (33) to invert first the Fourier transform and finally the Laplace transform.
}

cases of (i) a strongly anticorrelated process ( $\epsilon$ close to -1$)$, Fig. 1(a); (ii) a weakly anticorrelated process, Fig. 1(b); (iii) the independent case $(\epsilon=0)$, Fig. 1(c); (iv) a weakly correlated process, Fig. 1(d); (v) a strongly correlated process ( $\epsilon$ close to 1), Fig. 1(e); and finally, (vi) the completely persistent case $(\epsilon=1)$, Fig. 1(f). The visible effect of antipersistent memory in the process is that the probability density function becomes narrower around $x=0$. Thus, for larger negative values of $\epsilon$, the system tends to remain longer near the origin, and the process exhibits subdiffusive behavior for small time scales. In fact, from Eq. (32) we have for $\lambda t \ll 1$ that

$$
\sigma(t)=\sqrt{\left\langle X^{2}(t)\right\rangle} \approx \sqrt{\mu_{2} \lambda t+\epsilon \mu_{1}^{2} \lambda^{2} t^{2}},
$$

and therefore the process is subdiffusive for $\epsilon<0$, diffusive in the independent case (recall that this statement is valid for all time scales), and superdiffusive when $\epsilon>0$.

For large positive values of the correlation parameter $\epsilon$ [see Figs. 1(e) and 1(f)], the unconditional propagator presents another interesting property: there are two modes in the probability density function. These two modes are located in the vicinity of the conditional first moments $\left\langle X^{( \pm)}(t)\right\rangle$,

$$
\left\langle X^{( \pm)}(t)\right\rangle=\int_{-\infty}^{\infty} x p^{( \pm)}(x, t) d x= \pm \mu_{1} \frac{\epsilon}{1-\epsilon}\left(1-e^{-\lambda(1-\epsilon) t}\right),
$$

and become more and more noticeable for increasing values of $\epsilon$, as we show in Fig. 2(a). However, this apparent bimodality must disappear for large time scales, since if $\lambda t \gg 1$ we have $^{3}$

$$
\sigma(t) \sim \sqrt{\left(\mu_{2}+\frac{2 \mu_{1}^{2} \epsilon}{1-\epsilon}\right) \lambda t}
$$

whenever $\epsilon \neq 1$. This means that we must eventually attain the diffusive limit, as depicted in Fig. 2(b). Only in the completely persistent case $(\epsilon=1)$ is the phenomenon not of transient nature, because in this case the process is superdiffusive at all time scales:

$$
\sigma(t)=\sqrt{\mu_{2} \lambda t+\mu_{1}^{2} \lambda^{2} t^{2}}
$$

\section{A WEAKLY DEPENDENT MODEL}

Obtaining exact expressions for the propagator is usually quite involved, not to say impossible, in many practical situations, and we have to resort to approximations based on perturbation solutions of the integral equation (8). With this purpose in mind we will work with the following form of the joint density that generalizes the jump density given in Eq. (14):

$$
\rho\left(x^{\prime}, t^{\prime} \mid \xi, \tau\right)=\rho\left(x^{\prime}, t^{\prime}\right)\left[1+\epsilon g\left(x^{\prime}, t^{\prime} \mid \xi, \tau\right)\right],
$$

where $\rho(x, t)$ is the unconditional joint density satisfying

\footnotetext{
${ }^{3}$ Note that the expression inside the square root is positive definite even when $-1 \leqslant \epsilon<0$ because the Cauchy-Schwarz inequality implies that $\mu_{1}^{2}<\mu_{2}$ for $h\left(x^{\prime}\right) \neq\left[\delta\left(x^{\prime}-c\right)+\delta\left(x^{\prime}+c\right)\right] / 2$. The latter case corresponds to a process that moves back and forth between two fixed points, when $\epsilon=-1$.
} 


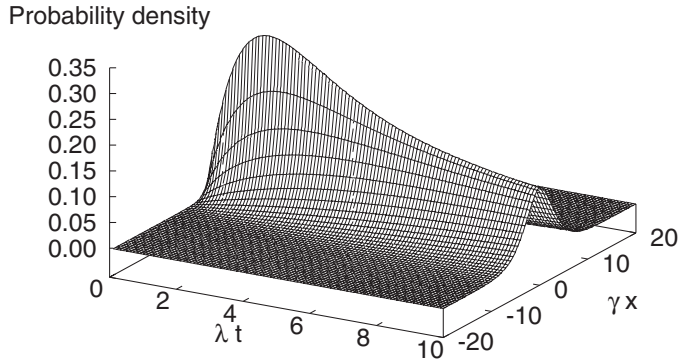

(a)

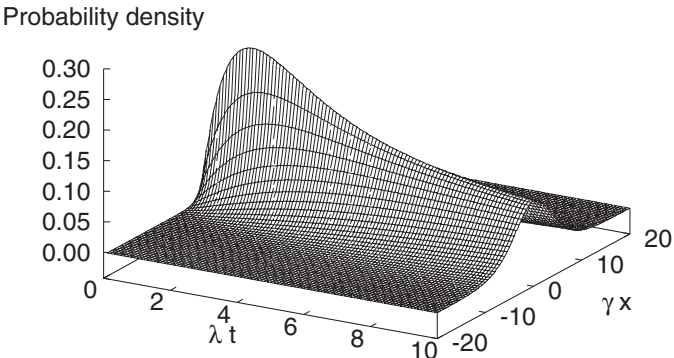

(c)

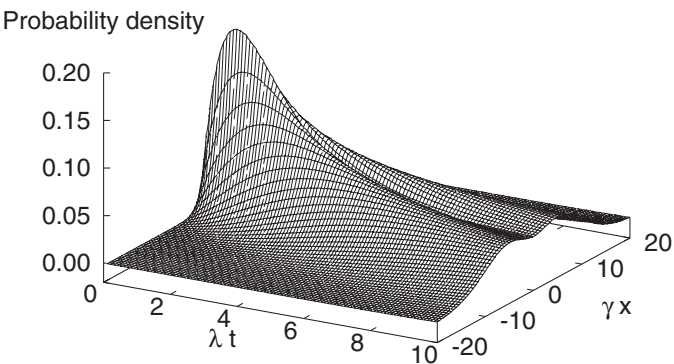

(e)

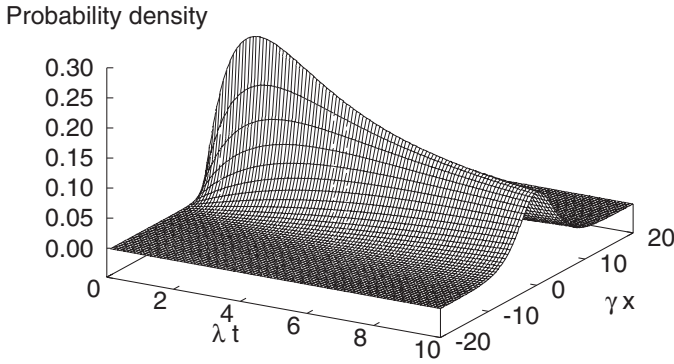

(b)

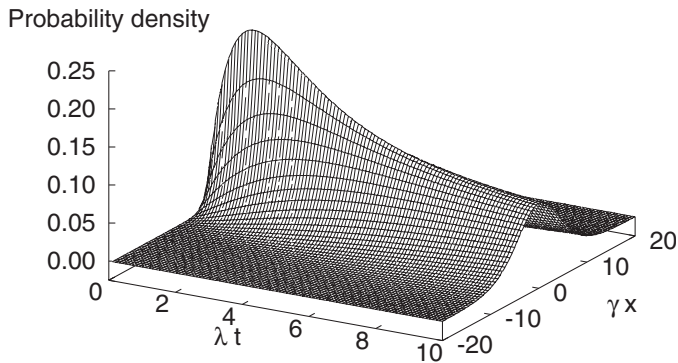

(d)

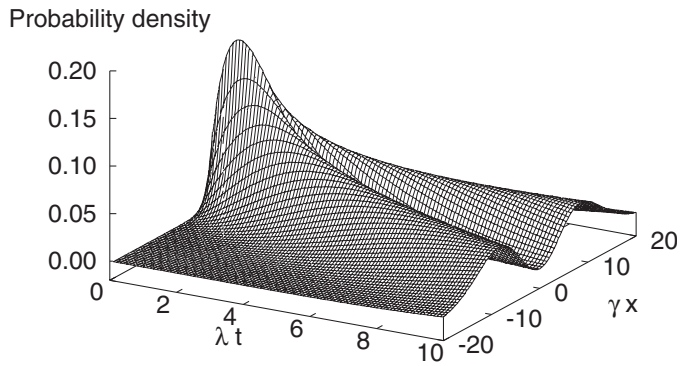

(f)

FIG. 1. Probability density function for different degrees of correlation: $\epsilon=$ (a) -0.8 , (b) -0.2 , (c) 0 , (d) 0.2 , (e) 0.8 , and (f) 1 . We can see how the negatively correlated processes concentrate the probability around the origin, whereas the positively correlated processes spread faster. Within this context, if the correlation is large enough, the system shows a transient bimodality.

$$
\rho\left(x^{\prime}, t^{\prime}\right)=\int_{0}^{\infty} d \tau \int_{-\infty}^{\infty} \rho\left(x^{\prime}, t^{\prime} \mid \xi, \tau\right) \rho(\xi, \tau) d \xi
$$

and the function $g\left(x^{\prime}, t^{\prime} \mid \xi, \tau\right)$ indicates the correlations between the waiting time and the jump of the current sojourn and those of the preceding sojourn. $\epsilon$ is a parameter measuring the strength of this correlation. In what follows we will suppose that $\epsilon$ is small, i.e., the model is weakly dependent. The function $g$ is not arbitrary and must satisfy two consistency conditions. Indeed, from the normalization of the densities $\rho\left(x^{\prime}, t^{\prime} \mid \xi, \tau\right)$ and $\rho\left(x^{\prime}, t^{\prime}\right)$,

$$
\int_{0}^{\infty} d t^{\prime} \int_{-\infty}^{\infty} \rho\left(x^{\prime}, t^{\prime} \mid \xi, \tau\right) d x^{\prime}=\int_{0}^{\infty} d t^{\prime} \int_{-\infty}^{\infty} \rho\left(x^{\prime}, t^{\prime}\right) d x^{\prime}=1
$$

it immediately follows that

$$
\int_{0}^{\infty} d t^{\prime} \int_{-\infty}^{\infty} \rho\left(x^{\prime}, t^{\prime}\right) g\left(x^{\prime}, t^{\prime} \mid \xi, \tau\right) d x^{\prime}=0 .
$$

On the other hand, plugging Eq. (37) into Eq. (38) and again taking into account normalization, we get

$$
\int_{0}^{\infty} d \tau \int_{-\infty}^{\infty} \rho(\xi, \tau) g\left(x^{\prime}, t^{\prime} \mid \xi, \tau\right) d \xi=0 .
$$

We also observe that, if $\rho\left(x^{\prime}, t^{\prime}\right) g\left(x^{\prime}, t^{\prime} \mid \xi, \tau\right)$ and $\rho(\xi, \tau) g\left(x^{\prime}, t^{\prime} \mid \xi, \tau\right)$ are integrable functions with respect to $t^{\prime}$ and $\tau$, respectively, then for the consistency conditions Eqs. (39) and (40) to hold it suffices that 

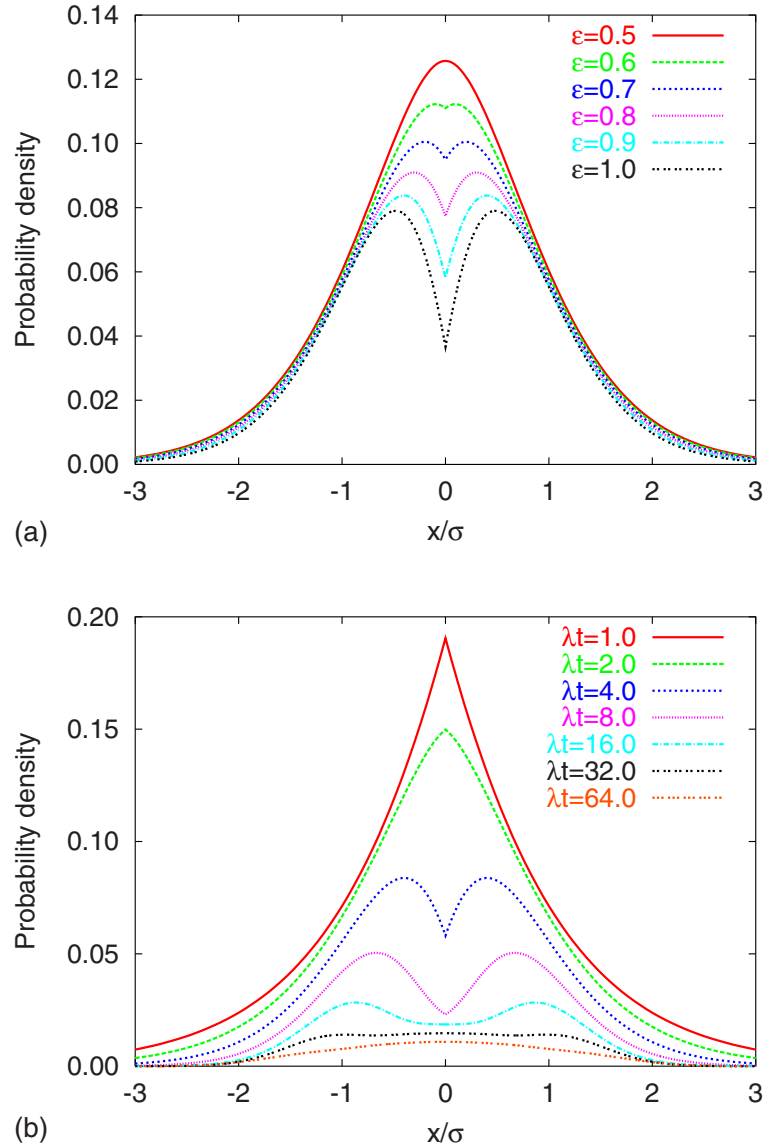

FIG. 2. (Color online) Roles of $\epsilon$ and $t$ in the bimodality of the probability density function. In (a) we can see how bimodality becomes more evident for larger values of $\epsilon$, when the value of $\lambda t$ $=4.0$ is kept fixed. In (b) we can check how this feature finally disappears even in a strongly correlated process, $\epsilon=0.9$. We have used the standard deviation of the process, $\sigma(t)$, in order to make the plots commensurable.

$$
\int_{-\infty}^{\infty} \rho\left(x^{\prime}, t^{\prime}\right) g\left(x^{\prime}, t^{\prime} \mid \xi, \tau\right) d x^{\prime}=\int_{-\infty}^{\infty} g\left(x^{\prime}, t^{\prime} \mid \xi, \tau\right) \rho(\xi, \tau) d \xi=0
$$

The starting point of our analysis is the renewal equation for the joint Fourier-Laplace transform of the propagator $\tilde{p}(\omega, s \mid \xi, \tau)$. Substituting then Eq. (37) into Eq. (11), we have

$$
\begin{aligned}
\tilde{p}(\omega, s \mid \xi, \tau)= & \widetilde{p}_{0}(\omega, s \mid \xi, \tau)+\int_{0}^{\infty} d t^{\prime} e^{-s t^{\prime}} \int_{-\infty}^{\infty} e^{i \omega x^{\prime}} \rho\left(x^{\prime}, t^{\prime}\right) \\
& \times\left[1+\epsilon g\left(x^{\prime}, t^{\prime} \mid \xi, \tau\right)\right] \widetilde{p}\left(\omega, s \mid x^{\prime}, t^{\prime}\right) d x^{\prime} .
\end{aligned}
$$

Assuming that $\epsilon$ is small, we look for a solution to this equation in the form

$$
\widetilde{p}(\omega, s \mid \xi, \tau)=\widetilde{q}(\omega, s)+\sum_{n=1}^{\infty} \epsilon^{n} \widetilde{p}^{(n)}(\omega, s \mid \xi, \tau) .
$$

In order to proceed further we need to know the dependence on $\epsilon$ of the propagator prior to the first sojourn. From Eqs. (9), (10), and (37), we write

$$
p_{0}(x, t \mid \xi, \tau)=\delta(x) \int_{t}^{\infty} d t^{\prime} \int_{-\infty}^{\infty} \rho\left(x^{\prime}, t^{\prime}\right)\left[1+\epsilon g\left(x^{\prime}, t^{\prime} \mid \xi, \tau\right)\right] d x^{\prime},
$$

but

$$
\int_{t}^{\infty} d t^{\prime} \int_{-\infty}^{\infty} \rho\left(x^{\prime}, t^{\prime}\right) d x^{\prime}=\Psi(t)
$$

and using the consistency condition (41) we finally get

$$
p_{0}(x, t \mid \xi, \tau)=\delta(x) \Psi(t) ;
$$

hence $p_{0}$ is independent of $\epsilon$.

One can easily see by substituting Eqs. (43) and (44) into Eq. (42) that the zeroth-order $\widetilde{q}(\omega, s)$ corresponds to the independent case,

$$
\widetilde{q}(\omega, s)=\frac{\hat{\Psi}(s)}{1-\widetilde{\rho}(\omega, s)},
$$

while for $n=1,2,3, \ldots$ we have the recursive integral equations

$$
\begin{aligned}
\widetilde{p}^{(n)}(\omega, s \mid \xi, \tau)= & Q^{(n-1)}(\omega, s \mid \xi, \tau)+\int_{0}^{\infty} d t^{\prime} e^{-s t^{\prime}} \int_{-\infty}^{\infty} e^{i \omega x^{\prime}} \rho\left(x^{\prime}, t^{\prime}\right) \\
& \times \widetilde{p}^{(n)}\left(\omega, s \mid x^{\prime}, t^{\prime}\right) d x^{\prime},
\end{aligned}
$$

where

$$
\begin{aligned}
Q^{(n-1)}(\omega, s \mid \xi, \tau)= & \int_{0}^{\infty} d t^{\prime} e^{-s t^{\prime}} \int_{-\infty}^{\infty} e^{i \omega x^{\prime}} \rho\left(x^{\prime}, t^{\prime}\right) g\left(x^{\prime}, t^{\prime} \mid \xi, \tau\right) \\
& \times \widetilde{p}^{(n-1)}\left(\omega, s \mid x^{\prime}, t^{\prime}\right) d x^{\prime}
\end{aligned}
$$

and

$$
\begin{aligned}
Q^{(0)}(\omega, s \mid \xi, \tau)= & \widetilde{q}(\omega, s) \int_{0}^{\infty} d t^{\prime} e^{-s t^{\prime}} \int_{-\infty}^{\infty} e^{i \omega x^{\prime}} \rho\left(x^{\prime}, t^{\prime}\right) \\
& \times g\left(x^{\prime}, t^{\prime} \mid \xi, \tau\right) d x^{\prime} .
\end{aligned}
$$

In the Appendix we show that the solution to Eq. (45) is given by the recursive expression $(n=1,2,3, \ldots)$

$$
\begin{aligned}
\widetilde{p}^{(n)}(\omega, s \mid \xi, \tau)= & \int_{0}^{\infty} d t^{\prime} e^{-s t^{\prime}} \int_{-\infty}^{\infty} e^{i \omega x^{\prime}} \rho\left(x^{\prime}, t^{\prime}\right) \\
& \times\left(g\left(x^{\prime}, t^{\prime} \mid \xi, \tau\right)+\frac{1}{1-\widetilde{\rho}(\omega, s)} G\left(x^{\prime}, t^{\prime} \mid \omega, s\right)\right) \\
& \times \widetilde{p}^{(n-1)}\left(\omega, s \mid x^{\prime}, t^{\prime}\right) d x^{\prime},
\end{aligned}
$$

where

$$
G\left(x^{\prime}, t^{\prime} \mid \omega, s\right) \equiv \int_{0}^{\infty} d \tau e^{-s \tau} \int_{-\infty}^{\infty} e^{i \omega \xi} \rho(\xi, \tau) g\left(x^{\prime}, t^{\prime} \mid \xi, \tau\right) d \xi
$$

and

$$
\widetilde{p}^{(0)}\left(\omega, s \mid x^{\prime}, t^{\prime}\right)=\widetilde{q}(\omega, s)=\frac{\hat{\Psi}(s)}{1-\widetilde{\rho}(\omega, s)} .
$$

For $n=1$ we have 


$$
\begin{aligned}
\widetilde{p}^{(1)}(\omega, s \mid \xi, \tau)= & \widetilde{q}(\omega, s) \int_{0}^{\infty} d t^{\prime} e^{-s t^{\prime}} \int_{-\infty}^{\infty} e^{i \omega x^{\prime}} \rho\left(x^{\prime}, t^{\prime}\right) \\
& \times\left(g\left(x^{\prime}, t^{\prime} \mid \xi, \tau\right)+\frac{1}{1-\widetilde{\rho}(\omega, s)}\right. \\
& \left.\times G\left(x^{\prime}, t^{\prime} \mid \omega, s\right)\right) d x^{\prime}
\end{aligned}
$$

which, after defining

$$
\widetilde{g}_{\rho}(\omega, s \mid \xi, \tau) \equiv \int_{0}^{\infty} d t^{\prime} e^{-s t^{\prime}} \int_{-\infty}^{\infty} e^{i \omega x^{\prime}} \rho\left(x^{\prime}, t^{\prime}\right) g\left(x^{\prime}, t^{\prime} \mid \xi, \tau\right) d x^{\prime}
$$

and [cf. Eq. (49)]

$$
\widetilde{G}_{\rho}(\omega, s) \equiv \int_{0}^{\infty} d t^{\prime} e^{-s t^{\prime}} \int_{-\infty}^{\infty} e^{i \omega x^{\prime}} \rho\left(x^{\prime}, t^{\prime}\right) G\left(x^{\prime}, t^{\prime} \mid \omega, s\right) d x^{\prime},
$$

can be written as

$$
\widetilde{p}^{(1)}(\omega, s \mid \xi, \tau)=\left(\widetilde{g}_{\rho}(\omega, s \mid \xi, \tau)+\frac{1}{1-\widetilde{\rho}(\omega, s)} \widetilde{G}_{\rho}(\omega, s)\right) \widetilde{q}(\omega, s) .
$$

Therefore, the joint Fourier-Laplace transform of the propagator up to first order in $\epsilon$ is

$$
\begin{aligned}
\tilde{p}(\omega, s \mid \xi, \tau)= & {\left[1+\epsilon\left(\tilde{g}_{\rho}(\omega, s \mid \xi, \tau)+\frac{\widetilde{G}_{\rho}(\omega, s)}{1-\tilde{\rho}(\omega, s)}\right)\right.} \\
& \left.+O\left(\epsilon^{2}\right)\right] \frac{\hat{\Psi}(s)}{1-\tilde{\rho}(\omega, s)} .
\end{aligned}
$$

From the above expression for the conditional propagator we can also get the unconditional propagator defined by

$$
p(x, t)=\int_{0}^{\infty} d \tau \int_{-\infty}^{\infty} \rho(\xi, \tau) p(x, t \mid \xi, \tau) d \xi .
$$

From Eq. (52) and taking into account the normalization of $\rho(\xi, \tau)$, we have

$$
\begin{aligned}
\tilde{p}(\omega, s)= & {\left[1+\epsilon\left(\int_{0}^{\infty} d \tau \int_{-\infty}^{\infty} \rho(\xi, \tau) \tilde{g}_{\rho}(\omega, s \mid \xi, \tau) d \xi\right.\right.} \\
& \left.\left.+\frac{\widetilde{G}_{\rho}(\omega, s)}{1-\tilde{\rho}(\omega, s)}\right)+O\left(\epsilon^{2}\right)\right] \frac{\hat{\Psi}(s)}{1-\tilde{\rho}(\omega, s)} .
\end{aligned}
$$

But from Eqs. (40) and (50) one can easily see that

$$
\int_{0}^{\infty} d \tau \int_{-\infty}^{\infty} \rho(\xi, \tau) \widetilde{g}_{\rho}(\omega, s \mid \xi, \tau) d \xi=0
$$

Hence

$$
\tilde{p}(\omega, s)=\left(1+\epsilon \frac{\tilde{G}_{\rho}(\omega, s)}{1-\tilde{\rho}(\omega, s)}+O\left(\epsilon^{2}\right)\right) \frac{\hat{\Psi}(s)}{1-\tilde{\rho}(\omega, s)} .
$$

We will finally present an instrumental example of the use of the perturbation technique just developed. We assume, as in Sec. IV, that the joint density $\rho$ factorizes as [cf. Eqs. (12) and (14)]

$$
\rho\left(x^{\prime}, t^{\prime} \mid \xi, \tau\right)=\psi\left(t^{\prime}\right) h\left(x^{\prime}\right)\left[1+\epsilon g\left(x^{\prime} \mid \xi\right)\right],
$$

where $|\epsilon| \ll 1$ and $h(\xi)$ is an even function of $\xi$. In contrast to the solvable case discussed in Sec. IV in which the correlation $g\left(x^{\prime} \mid \xi\right)$ depends solely on the signs of consecutive jumps [cf. Eq. (18)], we now assume that the correlation depends also on jump sizes. We thus suppose

$$
g\left(x^{\prime} \mid \xi\right)=\operatorname{sgn}\left(x^{\prime}\right) e^{-a\left(\left|x^{\prime}\right|+|\xi|\right)} \operatorname{sgn}(\xi)
$$

$(a \geqslant 0)$. With this correlation, we will evaluate the expressions for the propagators $p(x, t \mid \xi)$ and $p(x, t)$ as given, respectively, by Eqs. (52) and (54) for their joint FourierLaplace transform up to first order in $\epsilon$. To this end we need the explicit expressions for the auxiliary quantities $\widetilde{g}_{\rho}(\omega, s \mid \xi)$ and $\widetilde{G}_{\rho}(\omega, s)$ which appear in Eqs. (52) and (54).

Using Eqs. (55) and (56), the expression for $\widetilde{g}_{\rho}(\omega, s \mid \xi)$ defined in Eq. (50) can be written as

$$
\tilde{g}_{\rho}(\omega, s \mid \xi)=\hat{\psi}(s) \operatorname{sgn}(\xi) e^{-a|\xi|} \int_{-\infty}^{\infty} \operatorname{sgn}\left(x^{\prime}\right) e^{i \omega x^{\prime}-a\left|x^{\prime}\right|} h\left(x^{\prime}\right) d x^{\prime}
$$

But taking into account the symmetry of $h\left(x^{\prime}\right)$ expressed by Eq. (17), we can write

$$
\int_{-\infty}^{\infty} \operatorname{sgn}\left(x^{\prime}\right) e^{i \omega x^{\prime}-a\left|x^{\prime}\right|} h\left(x^{\prime}\right) d x^{\prime}=2 i \int_{0}^{\infty} e^{-a x^{\prime}} h\left(x^{\prime}\right) \sin \omega x^{\prime} d x^{\prime} .
$$

Hence

$$
\tilde{g}_{\rho}(\omega, s \mid \xi)=2 i \hat{\psi}(s) \tilde{h}_{\mathrm{s}}(\omega, a) e^{-a|\xi|} \operatorname{sgn}(\xi)
$$

where

$$
\tilde{h}_{\mathrm{s}}(\omega, a) \equiv \int_{0}^{\infty} e^{-a x^{\prime}} h\left(x^{\prime}\right) \sin \omega x^{\prime} d x^{\prime}
$$

is the Fourier sine transform of $e^{-a x^{\prime}} h\left(x^{\prime}\right)$.

Proceeding in an analogous way, we see that the expression for $\widetilde{G}_{\rho}(\omega, s)$ defined in Eq. (51) is given by

$$
\tilde{G}_{\rho}(\omega, s)=\hat{\psi}^{2}(s)\left(\int_{-\infty}^{\infty} \operatorname{sgn}\left(x^{\prime}\right) h\left(x^{\prime}\right) e^{i \omega x^{\prime}-a\left|x^{\prime}\right|} d x^{\prime}\right)^{2} .
$$

But as we have just seen [cf. Eq. (58)]

$$
\int_{-\infty}^{\infty} \operatorname{sgn}\left(x^{\prime}\right) h\left(x^{\prime}\right) e^{i \omega x^{\prime}-a\left|x^{\prime}\right|} d x=2 i \tilde{h}_{\mathrm{s}}(\omega, a)
$$

whence

$$
\tilde{G}_{\rho}(\omega, s)=-4 \hat{\psi}^{2}(s) \tilde{h}_{\mathrm{s}}^{2}(\omega, a) .
$$

Substituting Eqs. (57) and (59) into Eq. (52) yields 


$$
\begin{aligned}
\tilde{p}(\omega, s \mid \xi)= & {\left[1+\epsilon \hat{\psi}(s) \widetilde{h}_{\mathrm{s}}(\omega, a)\left(2 i e^{-a|\xi|} \operatorname{sgn}(\xi)\right.\right.} \\
& \left.\left.-\frac{4 \hat{\psi}(s) \tilde{h}_{\mathrm{s}}(\omega, a)}{1-\hat{\psi}(s) \tilde{h}(\omega)}\right)+O\left(\epsilon^{2}\right)\right] \frac{\hat{\Psi}(s)}{1-\hat{\psi}(s) \tilde{h}(\omega)} .
\end{aligned}
$$

For Poissonian sojourns [cf. Eq. (28)] we can take the inverse Laplace transform of this expression, which yields the conditional characteristic function

$$
\begin{aligned}
\tilde{p}(\omega, t \mid \xi)= & e^{-\lambda t[1-\tilde{h}(\omega)]} \\
& +\epsilon\left[2 i \frac{\widetilde{h_{\mathrm{s}}}(\omega, a)}{\widetilde{h}(\omega)}\left(-e^{-\lambda t}+e^{-\lambda t[1-\tilde{h}(\omega)]}\right) e^{-a|\xi|} \operatorname{sgn}(\xi)\right. \\
& -4\left(\frac{\tilde{h}_{\mathrm{s}}(\omega, a)}{\widetilde{h}(\omega)}\right)^{2}\left\{e^{-\lambda t}+[\lambda t \tilde{h}(\omega)-1] e^{-\lambda t[1-\tilde{h}(\omega)]\}}\right] \\
& +O\left(\epsilon^{2}\right) .
\end{aligned}
$$

The unconditional characteristic function can be analogously obtained through Eqs. (54) and (59) or else directly by substituting Eq. (60) into

$$
\widetilde{p}(\omega, t)=\int_{-\infty}^{\infty} \widetilde{p}(\omega, t \mid \xi) h(\xi) d \xi ;
$$

by either way one chooses, the final result is

$$
\begin{aligned}
\tilde{p}(\omega, t)= & e^{-\lambda t[1-\tilde{h}(\omega)]}-4 \epsilon\left(\frac{\widetilde{h}_{\mathrm{s}}(\omega, a)}{\widetilde{h}(\omega)}\right)^{2} \\
& \times\left\{e^{-\lambda t}+[\lambda t \tilde{h}(\omega)-1] e^{-\lambda t[1-\tilde{h}(\omega)]}\right\}+O\left(\epsilon^{2}\right) .
\end{aligned}
$$

For this example the variance of the process, $\left\langle X^{2}(t)\right\rangle=-\partial^{2} \widetilde{p}(\omega, s) /\left.\partial \omega^{2}\right|_{\omega=0}$, is easily seen to be

$$
\left\langle X^{2}(t)\right\rangle=\lambda t \mu_{2}+8 \epsilon \kappa_{a}^{2}\left(e^{-\lambda t}+\lambda t-1\right),
$$

where

$$
\mu_{2}=-\widetilde{h}^{\prime \prime}(0) \quad \text { and } \quad \kappa_{a}=\widetilde{h}_{\mathrm{s}}^{\prime}(0, a) .
$$

\section{SUMMARY AND CONCLUSIONS}

We have presented a generalization of the CTRW which includes correlations between consecutive sojourns and jumps. We have derived the general equations governing the time evolution of the dependent walk and have solved them exactly in some particular instances. We have also developed a general perturbation technique aimed at treating, within any desired degree of accuracy, weakly dependent models; that is, those models in which there is a low correlation between consecutive events.

Due to the extensive analytical apparatus and technical aspects contained in this paper, which may obscure the main objective and perhaps discourage potential users of the technique presented, we shall now summarize the key expressions of our development.
The model is based on a two-dimensional Markov series of jumps and sojourns, with a conditional joint density $\rho\left(\xi^{\prime}, \tau^{\prime} \mid \xi, \tau\right)$ defined in Eq. (7). The main objective of the CTRW is obtaining the so-called propagator $p(x, t \mid \xi, \tau)$, that is, the (conditional) probability density function of the process $X(t)$, provided we know the value of the last jump size $\xi$ and waiting time $\tau$. The propagator obeys a renewal equation [cf. Eq. (8)] and its Fourier-Laplace transform satisfies the integral equation (11). We have been able to find an exact solution to this equation when the joint density has the following form [cf. Eqs. (12) and (14)]:

$$
\rho\left(\xi^{\prime}, \tau^{\prime} \mid \xi, \tau\right)=\psi\left(\tau^{\prime}\right) h\left(\xi^{\prime}\right)\left[1+\epsilon \operatorname{sgn}\left(\xi^{\prime}\right) \operatorname{sgn}(\xi)\right],
$$

for which the correlation between jumps depends on whether they are increasing or decreasing, but not on their magnitude. In such a case the Fourier-Laplace transform of the unconditional propagator is given by [cf. Eq. (25)]

$$
\tilde{p}(\omega, s)=\frac{1-\epsilon \hat{\psi}(s) \widetilde{h}(\omega)}{1-(1+\epsilon) \hat{\psi}(s) \widetilde{h}(\omega)+4 \epsilon \hat{\psi}^{2}(s)|\tilde{H}(\omega)|^{2}} \hat{\Psi}(s) .
$$

In the case of Poissonian sojourns and Laplacian jumps we can invert this expression and obtain the propagator $p(x, t)$ for different values of $\epsilon$ [see Eqs. (34)-(36)]. From these expressions, we can see some interesting properties due to the existence of correlations such as the transitions from unimodal to bimodal distributions (see Figs. 1 and 2).

Although we have been able to solve Eq. (11) in the special case provided by Eq. (61), a general solution to the problem for any form of the joint density $\rho$ seems to be out of reach. However, in many practical situations the degree of dependence between current and past events is weak. In such cases it is possible to derive a perturbation technique which allows for an approximate solution to the above equation to any desired degree of accuracy. We thus write

$$
\rho\left(\xi^{\prime}, \tau^{\prime} \mid \xi, \tau\right)=\rho\left(\xi^{\prime}, \tau^{\prime}\right)\left[1+\epsilon g\left(\xi^{\prime}, \tau^{\prime} \mid \xi, \tau\right)\right],
$$

where $\rho\left(\xi^{\prime}, \tau^{\prime}\right)$ is the unconditional joint density, $g\left(\xi^{\prime}, \tau^{\prime} \mid \xi, \tau\right)$ indicates correlation, and $\epsilon$, now a small quantity, measures the strength of such a correlation. The function $g$ is not arbitrary and must obey some consistency conditions [cf. Eq. (41)] in order to keep the normalization of the $\rho$ 's.

The propagator can be written in the form of an infinite series,

$$
\widetilde{p}(\omega, s \mid \xi, \tau)=\widetilde{q}(\omega, s)+\sum_{n=1}^{\infty} \epsilon^{n} \widetilde{p}^{(n)}(\omega, s \mid \xi, \tau),
$$

where $\widetilde{q}(\omega, s)$ is the propagator when no correlation is present, that is, it corresponds to the propagator of the independent CTRW and is given by Eq. (6). The rest of the terms $\widetilde{p}^{(n)}(\omega, s \mid \xi, \tau)(n=1,2,3, \ldots)$ obey the integral equation (45) whose solution is given by Eq. (48), which allows us to compute $\widetilde{p}^{(n)}(\omega, s \mid \xi, \tau)$ if we know $\widetilde{p}^{(n-1)}(\omega, s \mid \xi, \tau)$. Obviously, by repeating this operation one can obtain $\widetilde{p}^{(n)}(\omega, s \mid \xi, \tau)$ for any $n=1,2,3, \ldots$ and, hence, an approximate expression for the propagator to any desired degree of accuracy, although, in many cases, the lowest order $n=1$ will 
suffice. In such a case the explicit expression for the propagator is [cf. Eqs. (50)-(52)]

$$
\begin{aligned}
\widetilde{p}(\omega, s \mid \xi, \tau)= & {\left[1+\epsilon\left(\tilde{g}_{\rho}(\omega, s \mid \xi, \tau)+\frac{\widetilde{G}_{\rho}(\omega, s)}{1-\widetilde{\rho}(\omega, s)}\right)+O\left(\epsilon^{2}\right)\right] } \\
& \times \frac{\hat{\Psi}(s)}{1-\widetilde{\rho}(\omega, s)},
\end{aligned}
$$

and for the unconditional propagator defined in Eq. (53) we have

$$
\tilde{p}(\omega, s)=\left(1+\epsilon \frac{\widetilde{G}_{\rho}(\omega, s)}{1-\widetilde{\rho}(\omega, s)}+O\left(\epsilon^{2}\right)\right) \frac{\hat{\Psi}(s)}{1-\widetilde{\rho}(\omega, s)} .
$$

We end this work by recalling that our first motivation to treat the problem of dependent CTRWs arose from our work in econophysics. In dealing with extreme time statistics of financial time series, in particular with the mean exit times of the process out of a given interval, we noticed that the observed behavior cannot be properly described by the traditional (i.e., independent) CTRW; one needs some degree of correlation between present and past events [25]. In spite of this specific motive, we certainly believe that a general development of the dependent CTRW - at least for a Markovian joint density - may be of broad interest because the independence assumption in the traditional CTRW is just a first approximation for many physical phenomena that are amenable to study within the CTRW framework [2,3]. In any case, in forthcoming presentations we will apply the method to financial time series.

\section{ACKNOWLEDGMENT}

The authors acknowledge partial support from Dirección General de Investigación under Contract No. FIS200605204.

\section{APPENDIX: SOLUTION TO A RECURSIVE INTEGRAL EQUATION}

We will solve the recursive integral equation (45):

$$
\begin{aligned}
\widetilde{p}^{(n)}(\omega, s \mid \xi, \tau)= & Q^{(n-1)}(\omega, s \mid \xi, \tau)+\int_{0}^{\infty} d t^{\prime} e^{-s t^{\prime}} \\
& \times \int_{-\infty}^{\infty} e^{i \omega x^{\prime}} \rho\left(x^{\prime}, t^{\prime}\right) \widetilde{p}^{(n)}\left(\omega, s \mid x^{\prime}, t^{\prime}\right) d x^{\prime},
\end{aligned}
$$

where $n=1,2,3, \ldots$. To this end we multiply both sides of Eq. (A1) by $e^{-s_{0} \tau+i \omega_{0} \xi} \rho(\xi, \tau)$ and integrate over $\xi$ and $\tau$. We obtain

$$
\widetilde{F}^{(n)}\left(\omega, s \mid \omega_{0}, s_{0}\right)=\widetilde{Q}^{(n-1)}\left(\omega, s \mid \omega_{0}, s_{0}\right)+\widetilde{\rho}\left(\omega_{0}, s_{0}\right) \widetilde{F}^{(n)}(\omega, s \mid \omega, s),
$$

where $\widetilde{\rho}\left(\omega_{0}, s_{0}\right)$ is the joint Fourier-Laplace transform of $\rho(\xi, \tau)$,

$$
\widetilde{F}^{(n)}\left(\omega, s \mid \omega_{0}, s_{0}\right) \equiv \int_{0}^{\infty} d \tau e^{-s_{0} \tau} \int_{-\infty}^{\infty} e^{i \omega_{0} \xi} \rho(\xi, \tau) \widetilde{p}^{(n)}(\omega, s \mid \xi, \tau) d \xi,
$$

and

$$
\begin{aligned}
\widetilde{Q}^{(n-1)}\left(\omega, s \mid \omega_{0}, s_{0}\right) \equiv & \int_{0}^{\infty} d \tau e^{-s_{0} \tau} \int_{-\infty}^{\infty} e^{i \omega_{0} \xi} \rho(\xi, \tau) \\
& \times Q^{(n-1)}(\omega, s \mid \xi, \tau) d \xi .
\end{aligned}
$$

Setting $\omega_{0}=\omega$ and $s_{0}=s$ in Eq. (A2), we get

$$
\widetilde{F}^{(n)}(\omega, s \mid \omega, s)=\frac{\widetilde{Q}^{(n-1)}(\omega, s \mid \omega, s)}{1-\widetilde{\rho}(\omega, s)},
$$

which introduced back into Eq. (A2) yields

$$
\begin{aligned}
\widetilde{F}^{(n)}\left(\omega, s \mid \omega_{0}, s_{0}\right)= & \widetilde{Q}^{(n-1)}\left(\omega, s \mid \omega_{0}, s_{0}\right) \\
& +\frac{\widetilde{\rho}\left(\omega_{0}, s_{0}\right)}{1-\widetilde{\rho}(\omega, s)} \widetilde{Q}^{(n-1)}(\omega, s \mid \omega, s) .
\end{aligned}
$$

From the definition of $\widetilde{F}^{(n)}\left(\omega, s \mid \omega_{0}, s_{0}\right)$ given in Eq. (A3), we see that the Fourier-Laplace inversion with respect to $\omega_{0}$ and $s_{0}$ of this quantity is

$$
\widetilde{F}^{(n)}\left(\omega, s \mid \omega_{0}, s_{0}\right) \rightarrow \rho(\xi, \tau) \widetilde{p}^{(n)}(\omega, s \mid \xi, \tau),
$$

and a similar expression holds for the inversion of $\widetilde{Q}^{(n-1)}\left(\omega, s \mid \omega_{0}, s_{0}\right)$. Therefore, the inversion of Eq. (A5) reads

$$
\widetilde{p}^{(n)}(\omega, s \mid \xi, \tau)=\widetilde{Q}^{(n-1)}(\omega, s \mid \xi, \tau)+\frac{1}{1-\widetilde{\rho}(\omega, s)} \widetilde{Q}^{(n-1)}(\omega, s \mid \omega, s) .
$$

By combining the definition of $\widetilde{Q}^{(n-1)}(\omega, s \mid \omega, s)$, given in Eq. (A4) when $\omega_{0}=\omega$ and $s_{0}=s$, with that of $Q^{(n-1)}(\omega, s \mid \xi, \tau)$ given in Eqs. (46) and (47), we write

$$
\begin{aligned}
\widetilde{Q}^{(n-1)}(\omega, s \mid \omega, s)= & \int_{0}^{\infty} d t^{\prime} e^{-s t^{\prime}} \int_{-\infty}^{\infty} e^{i \omega x^{\prime}} \rho\left(x^{\prime}, t^{\prime}\right) G\left(x^{\prime}, t^{\prime} \mid \omega, s\right) \\
& \times \widetilde{p}^{(n-1)}\left(\omega, s \mid x^{\prime}, t^{\prime}\right) d x^{\prime},
\end{aligned}
$$

where

$$
G\left(x^{\prime}, t^{\prime} \mid \omega, s\right) \equiv \int_{0}^{\infty} d \tau e^{-s \tau} \int_{-\infty}^{\infty} e^{i \omega \xi} \rho(\xi, \tau) g\left(x^{\prime}, t^{\prime} \mid \xi, \tau\right) d \xi
$$

Finally, substituting Eqs. (46) and (A7) into Eq. (A6), we obtain the recursive solution given in Eq. (48). 
[1] E. W. Montroll and G. H. Weiss, J. Math. Phys. 6, 167 (1965).

[2] E. W. Montroll and M. F. Shlesinger, in Nonequilibrium Phenomena II: From Stochastics to Hydrodynamics, edited by J. L. Lebowitz and E. W. Montroll (North-Holland, Amsterdam, 1984).

[3] G. H. Weiss, Aspects and Applications of the Random Walk (North-Holland, Amsterdam, 1994).

[4] B. Berkowitz and H. Scher, Phys. Rev. Lett. 79, 4038 (1997).

[5] M. Boguñá and Á. Corral, Phys. Rev. Lett. 78, 4950 (1997).

[6] E. Gudowska-Nowak and K. Weron, Phys. Rev. E 65, 011103 (2001).

[7] A. Helmstetter and D. Sornette, Phys. Rev. E 66, 061104 (2002)

[8] Á. Corral, Phys. Rev. Lett. 97, 178501 (2006).

[9] B. Berkowitz, G. Kosakowski, G. Margolin, and H. Sher, Ground Water 39, 593 (2001).

[10] M. Dentz and B. Berkowitz, Water Resour. Res. 39, 1111 (2003).

[11] P. Grigolini, L. Palatella, and G. Raffaelli, Fractals 9, 439 (2001).

[12] R. Kutner, Comput. Phys. Commun. 147, 565 (2002); Chem. Phys. 284, 481 (2002).

[13] E. Scalas, R. Gorenflo, and F. Mainardi, Physica A 284, 376 (2000); F. Mainardi, M. Raberto, R. Gorenflo, and E. Scalas, ibid. 287, 468 (2000).

[14] M. Raberto, E. Scalas, and F. Mainardi, Physica A 314, 749 (2002).

[15] E. Scalas, R. Gorenflo, and F. Mainardi, Phys. Rev. E 69,
011107 (2004).

[16] E. Scalas, Physica A 362, 225 (2006).

[17] R. Kutner and F. Switała, Quant. Finance 3, 201 (2003).

[18] J. Masoliver, M. Montero, and G. H. Weiss, Phys. Rev. E 67, 021112 (2003).

[19] P. Repetowicz and P. Richmond, Physica A 344, 108 (2004).

[20] J. Masoliver, M. Montero, J. Perelló, and G. H. Weiss, J. Econ. Behav. Organ. 61, 577 (2006).

[21] J. Masoliver, M. Montero, J. Perelló, and G. H. Weiss, Physica A 379, 151 (2007).

[22] D. R. Cox and H. D. Miller, The Theory of Stochastic Processes (Wiley, New York, 1965).

[23] G. Udny Yule, Philos. Trans. R. Soc. London, Ser. B 213, 21 (1925).

[24] W. Feller, An Introduction to Probability Theory and Its Applications (Wiley, New York, 1950).

[25] M. Montero, J. Perelló, J. Masoliver, F. Lillo, S. Miccichè, and R. N. Mantegna, Phys. Rev. E 72, 056101 (2005).

[26] Á. Corral, Tectonophysics 424, 117 (2006).

[27] P. L. Krapivsky and S. Redner, Am. J. Phys. 72, 591 (2004).

[28] G. E. Roberts and H. Kaufman, Table of Laplace Transforms (Sauders, Philadelphia, 1966).

[29] P. Gopikrishnan, V. Plerou, L. A. Nunes Amaral, M. Meyer, and H. E. Stanley, Phys. Rev. E 60, 5305 (1999); V. Plerou, P. Gopikrishnan, L. A. Nunes Amaral, M. Meyer, and H. E. Stanley, ibid. 60, 6519 (1999).

[30] A. C. Silva and V. M. Yakovenko, Physica A 382, 278 (2007). 